\title{
Ecological Study on Three Plantago Species and their Associates in Nile Delta Region
}

\author{
Ibrahim A. Mashaly, Ahmed M. Abd El-Gawad*, Mohamed E. Abu Ziada, Maha R. Deweeb \\ Botany Department, Faculty of Science, Mansoura University, Mansoura, 35516, Egypt
}

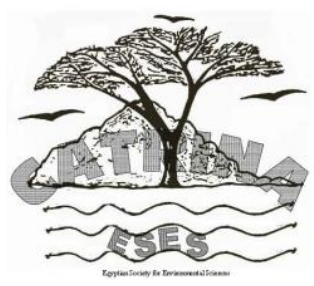

ABSTRACT
The present work aims at the following objectives: 1) vegetation analysis of the Plantago communities (P. major, P. lagopus, and P. squarrosa) in Nile Delta of Egypt using multivariate analysis, 2) analysis of variations in the edaphic variables controlling the abundance and distribution of the recognized plant communities, and 3) evaluation of the biodiversity of the Plantago communities in the study area. The sampled stands are distributed in four Governorates, namely: El-Dakahlia, Kafr El-Sheikh, El-Behira, and El-Sharkia. The plant cover and density of the recorded species were investigated in 60 sampled stands, and the vegetation classification and ordinated were achieved. Soil samples were collected and the physical and chemical properties were determined. The species richness and eveness in the three communities were aslo calculated. The application of Two-Way Indicator Species Analysis (TWINSPAN) classification based on the importance values of 105 plant species led to the recognition of four vegetation groups. Group A and B dominated by Plantago squarrosa. However, group C comprises dominated by Plantago lagopus. Group D dominated by Plantago major. The stand ordination is given by Detrended Correspondence Analysis (DCA) showed that the vegetation groups obtained by TWINSPAN classification are remarkable distinguishable and having a clear pattern of segregation on the ordination plane. Canonical Correspondence Analysis (CCA) exhibited that organic carbon, electrical conductivity, sand fraction, and $\mathrm{pH}$ value showed high significant corrections with the first and second axes. However, calcium carbonate, sodium, potassium and calcium cations as well as water-holding capacity, total dissolved phosphorus, silt and clay fractions exhibited a moderate significant correlation. $P$. squarrosa community was affected with many soil variables such as calcium carbonate, sodium, organic carbon, $\mathrm{pH}$ value, sand and magnesium. However, P. lagopus was affected by bicarbonates, potassium, sulfates and total nitrogen. Moreover, $P$. major showed a close relationship with electrical conductivity, water-holding capacity, total dissolved phosphorus, silt, clay, and porosity. The diversity measurements showed that the $P$. major community attained the highest richness and evenness, while the community of $P$. squarrosa showed the lowest diversity and eveness.

Keywords: Plantago, Nile Delta, soil factors, biodiversity, multivariate analysis.

\section{INTRODUCTION}

Weeds are plants growing where it not wanted and comprise the set of plant species found in agroecosystems. Weed species are well adapted to environments dominated by humans and have been associated with crop production since the origins of agriculture (Harlan, 1992). Weeds have many types depending on the habitat they invade such as agrestrals, ruderals, grassland weeds, water weeds, forestry weeds and environmental weeds (Holzner, 1982).

Weeds are commonly considered as unwanted intrudes into agro-ecosystems that compete for limited resources. In addition, weeds introduce negative impacts such as ecosystem effects that reduce crop yields, decrease animal growth, crop quality, increase their control costs or effect of survival, the growth of other species (Pimentel et al., 2000). On the other hand, weeds exhibit benefits that occur over a long time scale like increasing crop growth under certain conditions. Moreover, it provides a habitat for some beneficial insects or by providing habitat for natural enemies of pests decreasing the pest load on the crop resulting in increasing crop yield (Booth et al., 2003). In developing countries, farmers may spend 25 to 120 days handweeding a hectare of cropland (Akobundu, 1991) and still lose a quarter of the potential yield to weed competition (Parker and Fryer, 1975). Weeds can be viewed as valuable agroecosystem components that provide services complementing those obtained from crops. In India and Mexico, farmers consume Amaranthus, Brassica and Chenopodium species as nutritious foods before crop species are ready to harvest. Weed species can reduce soil erosion serve as important sources of fodder and medicine (Chikoye et al., 1995) and provide habitat for game birds and other desirable wildlife species (Sotherton et al., 1989 ). These types of beneficial effects indicate that weeds are not just agricultural pests, but can also play beneficial roles in agro-ecosystems.

Weed management has two principal objectives firstly, weed density should be reduced to tolerable levels, and secondly, the amount of damage that a given density of weeds inflicts on an associated crop should be reduced. The negative effect of weeds on crops can be limited not only by reducing weed density, but also by minimizing the resource consumption, growth, and competitive ability of each surviving weed (Mortensen et al., 1998).

The composition of weed communities should be shifted toward less aggressive, easier to manage species. Weed species differ in the amount of damage they inflict on crops and the degree of difficulty they impose on crop management and harvesting activities. Consequently, it is desirable to tip the balance of weed community composition from dominance by noxious species toward a preponderance of species that crops, livestock, and farmers can better tolerate. This can be

* Corresponding authore-mail: dgawad84@mans.edu.eg or dgawad84@yahoo.com 
achieved by selectively and directly suppressing undesirable weed species while manipulating environmental conditions to prevent their re-establishment (Sheley et al., 1996; Staver et al., 1995). Selective vegetation management is particularly well suited to agroecosystems dominated by perennial plants, such as orchards, pastures, and rangelands. Since the 1980s there has been increasing recognition that herbicides, applied in the course of normal farming practices, have contaminated surface and groundwater in many agricultural regions (Fuhrer, 1999; Larson et al., 1999). For weed management purposes, allelopathy is considered a strategy of control (Zimdahl, 2013).

In Egypt, a lot of studies were achived to characterize various weed communities. Mashaly et al. (2011) studied the weed vegetation-soil relationship in the Deltaic Mediterranean coast of Egypt. Abd El-Ghani and Amer (1990) studied the weed assemblages associated with broad bean fields in Monofiyia Governorate. Shaltout and El Fahar (1991) evaluated the species diversity and phenological behavior of the weed communities associated with common crops in the Nile delta regions. Shaltout et al. (1992) depicted the weed communities associated with common crops in the Nile delta region. Mashaly et al. (2011) investigated the ecology of weeds and invasive plant species in newly reclaimed areas in the Nile Delta.

Plantago in Egypt comprises 22 species (Boulos, 2009). These species have wide ecological amplitude. They are weeds of both arable lands and grasslands (Ghdifan et al., 2011; Mohsenzadeh et al., 2008). Some species of Plantago are used as traditional medicinal plants for centuries for various purposes, such as wound healing. They were reported to have biological activities including anti-inflammatory, analgesic, anti-tumoral, anti-spasmodic, hepatoprotective, antiviral, antibacterial, antifungal and antiulcerogenic (Abd Razik et al., 2012; Harput et al., 2012; Samuelsen, 2000). The present study aimed to analyze the vegetation composition of the three Plantago communities ( $P$. major L., P. lagopus L., and P. squarrosa Murray) in the Nile Delta of Egypt using multivariate analysis, as well as investigate the plant diversity of these communities. In addition, determine the soil factors controlling the abundance and distribution of the recognized plant communities in the study area.

\section{MATERIALS AND METHODS}

\section{Study Area}

The study area is located in Nile Delta which covers a total area of 2.25 million ha and is characterized by alluvial soils (clay to loamy). The Nile is the main source of water for irrigation, while the new land is located mainly on both the east and west sides of the Delta and scattered over various areas in the country (Fig. 1). The climate of the study area is arid, with a mean temperature of $12^{\circ} \mathrm{C}$ in winter and $26.5^{\circ} \mathrm{C}$ in summer. The annual rainfall ranges from 91.6 to 175.2 $\mathrm{mm}$. Mean relative humidity is lower in summer $(65 \%)$ than in winter $(81 \%)$ and evaporation is higher in summer (7.8 mm Piche/day) than in winter $(2.8 \mathrm{~mm}$ Piche/day) (Anonymous, 1977).

\section{Vegetation Analysis}

The sampled stands were distributed in the northern and central sections of the Nile Delta (Fig. 1). The stands representing $P$. lagopus $\mathrm{L}$. community were sampled in Gnakleas District (El-Behira Governorate), while the stands of $P$. major $\mathrm{L}$. community were represented in Talkha, El-Mansoura and Bilqas Districts (ElDakahlia Governorate) and El-Salhia District (ElSharkia Governorate). However, the sampled stands of $P$. squarrosa Murray community were designed in Idko and Rosetta Districts (El-Behira Governorate), Qalabshu village (El-Dakahlia Governorate) and Baltim District (Kafr El-Sheikh Governorate). After regular field visits to the different sites of the study area, 60 stands $(2 \times 5 \mathrm{~m}$ each, according to the minimal area) were designed for sampling the vegetation types in the different habitats of the study area. The chosen stands were distributed in the study area to cover all local physiographic variations within each habitat type and to ensure sampling of a wide range of vegetation variations.

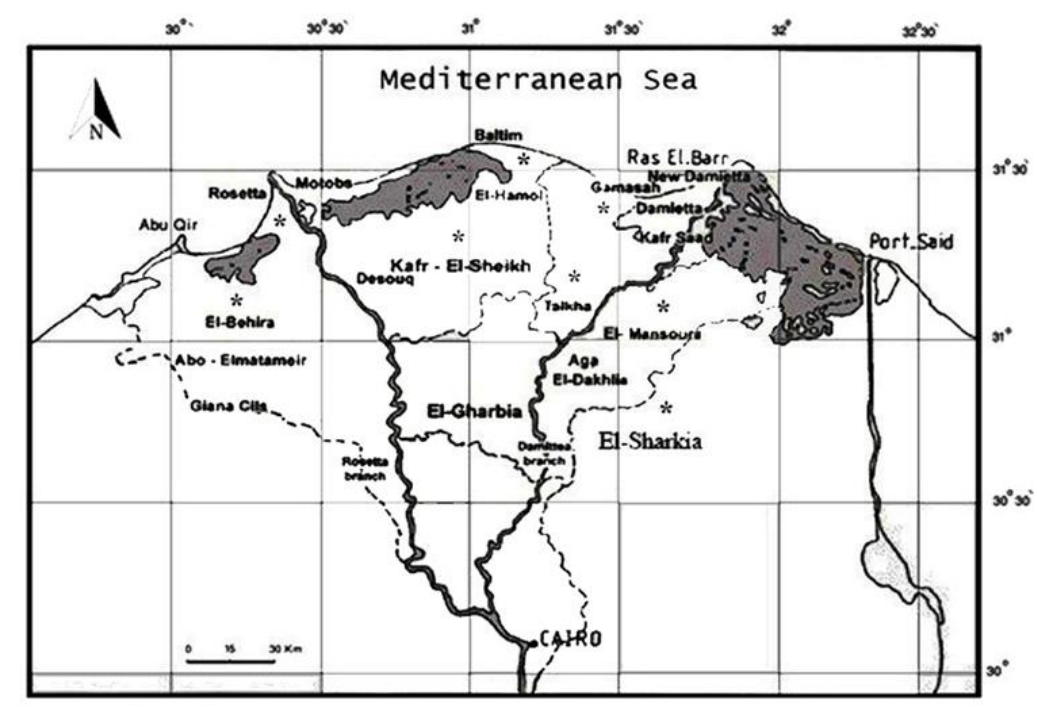

Figure (1): Map of the Nile Delta showing the locations of the sampled sites $\left(^{*}\right)$. 
The density and plant cover of each species were estimated in each sampled stand. The relative density was calculated by calculating the number of individual species relative to the total number of species in the sampled stand (Shukla and Chandel, 1989). However, plant cover of each species in the surveyed stands was measured using the line-intercept method (Canfield, 1941). The lengths of intercept of each species in a stand were measured in centimeters. These lengths were then summed and expressed as the relative value of the total length of all lines. Relative values of density and cover were summed up to give an estimate of its importance value (IV) in each stand, which is out of 200. The identification of the recorded species was following Tackholm (1974) and up to date by Boulos (1999-2005).

\section{Plant Diversity Measurements}

Two common diversity indices are Simpson's index and the Shannon-Wiener. Both the Simpson's and the Shannon-Wiener indices referred for richness and are non-parametric measures of species heterogeneity that makes no assumption about the normality of species abundance curve (Magurran, 1988). The following equation is using to calculate the Shannon-Wiener Diversity Index $(\mathrm{H})$ :

$$
H=\sum_{i=1}^{s} P_{i} \ln \left(P_{i}\right)
$$

Where: $P_{i}$ is the number of individuals of species $(s) i$ / total number of samples, and $S$ is the numbers of species encountered. While Shannon-Evenness Index (E) was calculated as follows:

$$
E=\frac{H^{\prime}}{\ln _{s}}
$$

However, the Simpson's Index (D) was determined according to the following equation:

$$
D=\frac{\sum_{i}\left[n_{i} \times\left(n_{i}-1\right)\right]}{[N \times(N-1)]}
$$

Where, $\mathrm{n}=$ the number of individuals of each different species; $\mathrm{N}=$ the total number of individuals of all the species.

\section{Soil Analysis}

Soil samples were collected from each stand (triplicates) representing a profile at a depth of 0-50 cm. Soil texture, water holding capacity (WHC), soil porosity, organic carbon, and sulfates were determined according to Piper (1947). Calcium carbonate content was determined by titration against $1 \mathrm{~N} \mathrm{NaOH}$ and expressed as a percentage to Jackson (1962). The soil solution (1:5) was prepared for each soil sample. The electrical conductivity, $\mathrm{pH}$, and chloride were determined by the method adopted by to Jackson (1962). Bicarbonates were determined by titration using $0.1 \mathrm{~N} \mathrm{HCl}$ (Pierce et al., 1958). Total dissolved phosphorus was determined by digestion and followed by direct stannous chloride method as described in American Public Health Association (APHA, 1998). The total nitrogen was determined by the conventional semi-micro modification of Kjeldahl method (Pirie, 1955). The extractable cations $\mathrm{Na}^{+}$and $\mathrm{K}^{+}$contents were determined using Flame Photometer (Model PHF 80 Biologie Spectrophotometer), while $\mathrm{Ca}^{2+}$ and $\mathrm{Mg}^{2+}$ were estimated using atomic absorption spectrometer (A Perkin-Elmer, Model 2380.USA) (Allen et al., 1974).

\section{Treatment of Data}

Two trends of multivariate analysis were applied in the present study (ordination and classification). Both trends have their merits in helping to understand the vegetation and environmental phenomena. The classification techniques applied here were the Two-Way Indicator Species Analysis (TWINSPAN) and Detrended Correspondence Analysis (DECORANA) (Hill 1979; Gauch and Whittaker 1981). TWINSPAN was carried out using Community Analysis Package (CAP) program (Henderson and Seaby 1999). However, the ordination techniques applied were the Detrended Correspondence Analysis (DCA) and the Canonical Correspondence Analysis (CCA) using CANOCO (ter Braak, 1987). The relationships between vegetation groups and edaphic variable can be indicated on the ordination diagram produced by Canonical Correspondence Analysis (CCA biplot), in which points represent plant species and arrows represent environmental variables. The simple linear correlation coefficient (r) was calculated to assess the relationships between the spatial variation in edaphic variables and vegetation measurements of the plant species (ordination axis).

Mean values and coefficient of variation of the plant species were calculated for the importance value of the plant species which was recorded in the stands representing the different vegetation groups in the major habitat types of the study area. Also, mean and standard errors were calculated for the soil variables. All statistical treatments applied here were according to Snedecor and Cochran (1968). The data for soil variables in relation to Plantago communities, as well as soil variables in relation to vegetation groups were subjected to ANOVA, where mean values were separated on the basis of Least Significant Difference (LSD) at 0.05 probability level.

\section{RESULTS}

\section{Vegetation Analysis (Classification and ordination of stands)}

The application of TWINSPAN classification based on the importance values of 105 plant species recorded in 60 sampled stands representing different habitat types of the study area, led to the recognition of four vegetation groups (Fig. 2 and Table 1). Group A comprises 3 stands dominated by Plantago squarrosa which has the highest importance value of this group $(\mathrm{IV}=81.7)$; the other important species were Moltkiopsis ciliata $(\mathrm{IV}=26.1)$, Stipagrostis lanata $(\mathrm{IV}=18.1)$ and Echinops spinosus (IV=16.2). Group B includes 17 stands dominated also by Plantago squarrosa $(\mathrm{IV}=53.9)$; the other important which attained relatively high importance values in this group were Erodium laciniatum (IV=23.7), Aegilops bicornis (IV=17.1), Echinops spinosus (IV=14.4) and Bromus catharactius (IV=5.8). Group C comprises 21 stands dominated by Plantago lagopus $(\mathrm{IV}=36.3)$. Cynodon dactylon $(\mathrm{IV}=21.5)$, 
Lolium multiflorum (IV=14.5), Emex spinosa (IV=13.2) Chenopodium murale (IV=10.3), Raphanus raphenistrum (IV=5.4) and Urospermum picroides ( IV=3.9) were the important species in this group. Group D comprises 19 stands dominated by Plantago major
(IV=39.3); the other important species were Rumex dentatus (IV=30.4), Cynodon dactylon (IV=16.2), Sonchus oleraceus L. (IV=14.7) and Malva parviflora $(\mathrm{IV}=14.3)$. On the other hand, Lotus glaber $(\mathrm{IV}=2.9)$ was identified as an indicator species in this group.

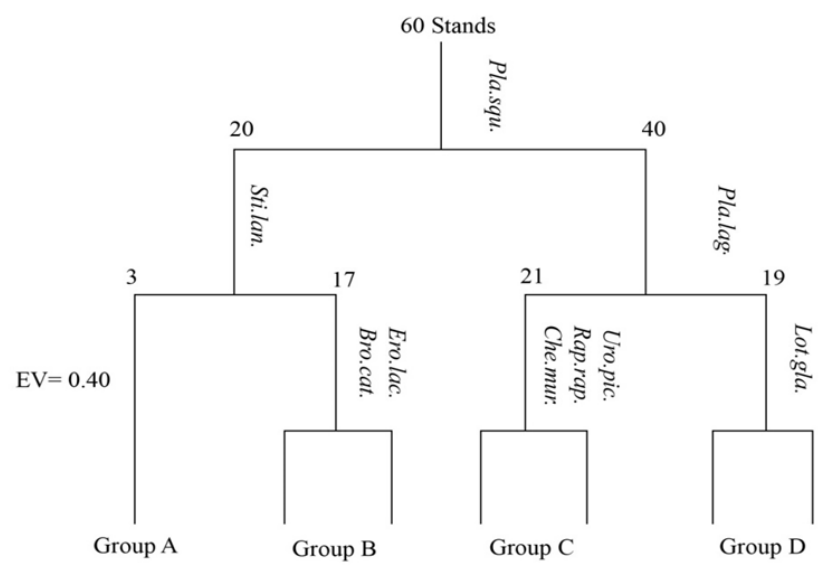

Figure (2): TWINSPAN dendrogram of 60 sampled stands based on the importance values of 105 species. Pla.squ.: Plantago squarrosa, Pla.lag.: Plantago lagopus, Sti.lan.: Stipagrostis lanata, Lot.gla.: Lotus glaber, Uro.pic.: Urospermum picroides, Rap.rap.: Raphanus raphanistrum, Che.mur.: Chenopodium murale, Ero.lac.: Erodium laciniatum, Bro.cat.: Bromus catharticus, and EV: Eigenvalue.

Table (1): Mean value and coefficient of variation (value between brackets) of the importance values (out of 200) of the recorded species in the different vegetation groups resulting from TWINSPAN classification.

\begin{tabular}{|c|c|c|c|c|}
\hline Species & Group A & Group B & Group C & Group D \\
\hline Aegilops bicornis (Forssk.) Jaub.\& Spach & $0.56(1.73)$ & $17.06(1.33)$ & -- & -- \\
\hline Alhagi graecorum Boiss. & -- & $5.99(3.01)$ & -- & -- \\
\hline Amaranthus lividus $\mathrm{L}$. & -- & -- & $0.45(4.58)$ & -- \\
\hline Ammannia baccifera $\mathrm{L}$. & -- & -- & -- & $0.82(4.36)$ \\
\hline Anagallis arvensis $\mathrm{L}$. & -- & -- & $0.82(2.75)$ & $1.83(2.87)$ \\
\hline Anchusa humilis (Desf.) I.M. Johnst. & -- & $1.70(3.30)$ & -- & -- \\
\hline Avena fatua $\mathrm{L}$. & -- & -- & $3.98(3.13)$ & -- \\
\hline Beta vulgaris $\mathrm{L}$. & -- & -- & -- & $3.22(1.80)$ \\
\hline Bidens pilosa $\mathrm{L}$. & -- & -- & $0.31(4.58)$ & $2.30(3.64)$ \\
\hline Brassica tournefortii Gouan & -- & $1.37(2.48)$ & $3.53(2.18)$ & -- \\
\hline Bromus catharticus Vahl & $13.13(0.88)$ & $5.48(2.48)$ & $1.67(2.48)$ & $1.42(3.04)$ \\
\hline Bromus diandrus Roth & -- & $0.32(4.12)$ & $3.50(3.47)$ & -- \\
\hline Cakile maritima Scop.subsp aegyptiaca (Wild.) Nyman & -- & $1.31(4.12)$ & -- & -- \\
\hline Calendula arvensis $\mathrm{L}$. & -- & -- & $0.41(3.27)$ & -- \\
\hline $\begin{array}{l}\text { Calligonum polygonoides L. subsp comosum (L'Her.) } \\
\text { Soskov }\end{array}$ & -- & $1.26(4.12)$ & -- & -- \\
\hline Carduus getulus Pomel & -- & $0.31(4.12)$ & -- & -- \\
\hline Carthamus tenuis (Boiss. \& Blanche) Bornm. & -- & $1.54(3.62)$ & $0.52(3.35)$ & -- \\
\hline Cenchrus biflorus Roxb. & -- & -- & $0.98(2.13)$ & -- \\
\hline Chenopodium album $\mathrm{L}$. & -- & -- & $1.86(2.46)$ & $0.54(4.36)$ \\
\hline Chenopodium giganteum D. Don & -- & -- & $0.43(4.58)$ & -- \\
\hline Chenopodium murale $\mathrm{L}$. & -- & -- & $10.28(1.08)$ & $5.24(1.42)$ \\
\hline Convolvulus arvensis $\mathrm{L}$. & -- & -- & $3.53(1.59)$ & $1.59(2.53)$ \\
\hline Conyza aegyptiaca (L.) Dryand. & -- & $0.75(2.93)$ & $0.07(4.58)$ & $0.14(4.36)$ \\
\hline Conyza bonariensis (L.) Cronquist & -- & -- & $1.47(2.16)$ & -- \\
\hline Coronopus didymus (L.) Sm. & -- & -- & $0.09(4.58)$ & -- \\
\hline Coronopus squamatus (Forssk.) Asch. & -- & -- & $0.37(4.58)$ & $1.12(3)$ \\
\hline Cutandia memphitica (Spreng.) Benth. & -- & $0.93(4.12)$ & -- & -- \\
\hline Cynodon dactylon (L.) Pers. & -- & $6.24(1.56)$ & $21.54(0.95)$ & $16.23(1.21)$ \\
\hline Cyperus alopecuroides Rottb. & -- & -- & -- & $0.33(4.36)$ \\
\hline Cyperus capitatus Vand. & $7.07(1.73)$ & $10.67(1.79)$ & -- & -- \\
\hline Cyperus rotundus $\mathrm{L}$. & -- & -- & $8.22(2.11)$ & -- \\
\hline Daucus litoralis $\mathrm{Sm}$. & $4.55(1.03)$ & $1.64(2.83)$ & -- & -- \\
\hline Echinochloa stagnina (Retz.) P. Beauv. & -- & -- & -- & $0.78(4.36)$ \\
\hline Echinops spinosus $L$. & $16.17(1.15)$ & $14.40(1.51)$ & -- & -- \\
\hline Echium angustifolium Mill. subsp sericum (Vahl) Koltz & -- & $9.0(1.95)$ & $0.98(3.19)$ & -- \\
\hline Eclipta prostrata $\mathrm{L}$. & -- & -- & -- & $0.97(4.36)$ \\
\hline Elymus farctus (Viv.) Runemark ex Melderis & $14.23(1.73)$ & -- & -- & - \\
\hline Emex spinosa (L.) Campd. & -- & -- & $13.19(0.99)$ & -- \\
\hline Erodium laciniatum (Cav.) Willd. & $11.48(0.94)$ & $23.69(1.13)$ & -- & -- \\
\hline
\end{tabular}


Mashaly et al.

\begin{tabular}{|c|c|c|c|c|}
\hline Species & Group A & Group B & Group C & Group D \\
\hline Euphorpia peplus L. & -- & -- & $1.47(4.58)$ & -- \\
\hline Euphorbia prostrata Aiton & -- & -- & $7.18(1.42)$ & $3.38(2.69)$ \\
\hline Euphorbia terracina $\mathrm{L}$. & -- & -- & -- & $0.49(4.36)$ \\
\hline Fumaria bracteosa Pomel & -- & -- & $0.35(3.28)$ & -- \\
\hline Hordeum murinum $\mathrm{L}$. & -- & $2.12(4.12)$ & $3.41(3.86)$ & -- \\
\hline Ifloga spicata (Forssk.) Sch. Bip. & -- & $0.62(4.12)$ & -- & -- \\
\hline Imperata cylindrica (L.) Raeusch. & -- & $2.71(4.12)$ & $11.03(1.52)$ & \\
\hline Lactuca serriola $\mathrm{L}$. & -- & -- & $0.48(2.84)$ & -- \\
\hline Lamium amplexicaule $\mathrm{L}$. & -- & -- & -- & $1.70(3.12)$ \\
\hline Launaea fragilis (Asso) Pau & -- & $0.15(4.12)$ & $0.39(4.58)$ & -- \\
\hline Launaea mucronata (Forssk.) Muschl. & $1.57(1.73)$ & $1.87(3.29)$ & -- & -- \\
\hline Lolium multiflorum Lam. & -- & -- & $14.46(1.18)$ & -- \\
\hline Lolium perenne $\mathrm{L}$. & -- & -- & $3.90(2.13)$ & $0.70(4.36)$ \\
\hline Lotus creticus L. & $4.00(1.73)$ & -- & -- & -- \\
\hline Lotus glaber Mill. & -- & -- & -- & $2.91(3.01)$ \\
\hline Lotus halophilus Boiss. \& Spruner & -- & $5.16(1.47)$ & -- & -- \\
\hline Malva parviflora $\mathrm{L}$. & -- & -- & $5.60(2.13)$ & $14.25(1.54)$ \\
\hline Medicago intertexta (L.) Mill. & -- & -- & -- & $0.42(4.36)$ \\
\hline Medicago polymorpha $\mathrm{L}$. & -- & -- & -- & $0.89(4.36)$ \\
\hline Melilotus indicus (L.) All. & -- & $0.08(4.12)$ & $4.65(1.93)$ & $0.72(3.81)$ \\
\hline Mentha longfolia (L.) Huds. & -- & -- & -- & $6.0(2.56)$ \\
\hline Mesembryanthemum crystallinum $\mathrm{L}$. & -- & $2.39(4.02)$ & -- & -- \\
\hline Moltkiopsis ciliata (Forssk.) I. M. Johnst. & $26.08(0.88)$ & -- & -- & -- \\
\hline Ononis serrata Forssk. & -- & $4.96(1.84)$ & -- & -- \\
\hline Oxalis corneculata $\mathrm{L}$. & -- & -- & -- & $3.31(2.99)$ \\
\hline Pancratium maritimum $\mathrm{L}$. & $0.43(1.73)$ & -- & -- & -- \\
\hline Paronychia arabica (L.) DC. & -- & $0.62(4.12)$ & -- & -- \\
\hline Pasplidium geminatum (Forssk.) Stapf & -- & -- & -- & $1.26(4.36)$ \\
\hline Pennisetum setaceum (Forssk.) Chiov. & -- & -- & -- & $4.69(3.21)$ \\
\hline Persicaria salicifolia (Brouss.exWilld.) Assenov & -- & -- & -- & $1.49(3.95)$ \\
\hline Phalaris minor Retz. & -- & -- & -- & $0.87(4.36)$ \\
\hline Phragmites australis (Cav.) Trin. ex Steud. & -- & -- & $0.50(4.58)$ & $3.64(4.36)$ \\
\hline Phyla nodiflora (L.) Greene & -- & -- & -- & $2.29(4.36)$ \\
\hline Picris asplenioides L. & $0.63(1.73)$ & $0.64(4.12)$ & -- & -- \\
\hline Plantago lagopus $\mathrm{L}$. & -- & -- & $36.32(0.90)$ & \\
\hline Plantago major L. & -- & -- & $4.33(4.58)$ & $39.25(0.75)$ \\
\hline Plantago squarrosa Murray & $81.72(0.18)$ & $53.94(0.58)$ & -- & -- \\
\hline Pоа аппиа $\mathrm{L}$. & -- & -- & -- & $0.43(3.08)$ \\
\hline Polygonum equisetiforme $\mathrm{Sm}$. & -- & -- & $0.94(3.51)$ & -- \\
\hline Polypogon monspeliensis (L.) Desf. & -- & -- & $0.41(4.58)$ & $2.63(3.88)$ \\
\hline Polypogon viridis (Gouan) Breistr. & -- & -- & -- & $0.18(4.36)$ \\
\hline Pseudorlaya pumila (L.) Grande & -- & $0.35(2.99)$ & -- & -- \\
\hline Ranunculus scleratus L. & -- & -- & -- & $0.48(4.36)$ \\
\hline Raphanus raphanistrum $\mathrm{L}$. & -- & -- & $5.41(2.24)$ & -- \\
\hline Reicharadia tingitana (L.) Roth & -- & $0.33(4.12)$ & -- & -- \\
\hline Rorippa palustris (L.) Besser & -- & -- & -- & $2.46(2.64)$ \\
\hline Rumex dentatus $\mathrm{L}$. & $0.28(1.73)$ & -- & $1.30(4.01)$ & $30.39(0.92)$ \\
\hline Rumex pictus L. & -- & $15.87(0.91)$ & -- & -- \\
\hline Schoenus nigricans $\mathrm{L}$. & -- & $0.51(4.12)$ & -- & -- \\
\hline Senecio glaucus $\mathrm{L}$. & -- & $3.59(2.11)$ & $0.59(1.85)$ & -- \\
\hline Sida alba $\mathrm{L}$ & -- & -- & $0.29(4.58)$ & $0.32(4.36)$ \\
\hline Silene succulenta Forssk. & -- & $0.15(4.12)$ & -- & -- \\
\hline Silene vivianii Steud. & -- & $0.08(4.12)$ & -- & -- \\
\hline Sisymbrium irio L. & -- & -- & $2.03(2.99)$ & -- \\
\hline Solanum nigrum $\mathrm{L}$. & -- & -- & $0.56(2.35)$ & $0.39(4.36)$ \\
\hline Sonchus oleraceus L. & -- & -- & $2.56(1.15)$ & $14.64(1.27)$ \\
\hline Sorghum virgatum (Hack.) Stapf & -- & -- & -- & $0.54(4.36)$ \\
\hline Stellaria pallida (Dumort.)Murb. & -- & -- & $0.40(3.96)$ & $10.01(1.49)$ \\
\hline Stipagrostis lanata (Forssk.)De Winter & $18.09(0.67)$ & $0.20(4.12)$ & -- & -- \\
\hline Symphyotrichum squamatum (Spreng.) Nesom & -- & -- & $0.63(4.58)$ & -- \\
\hline Torilis arvensis (Huds.) Link & -- & -- & $2.33(2.18)$ & $1.28(2.87)$ \\
\hline Urospermum picroides (L.) F. W. Schmidt & -- & -- & $3.92(1.26)$ & $1.91(4.36)$ \\
\hline Urtica urens L. & -- & -- & $4.02(2.45)$ & $1.08(4.36)$ \\
\hline Veronica anagallis-aquatica $\mathrm{L}$. & -- & -- & -- & $4.61(3.53)$ \\
\hline Vicia sativa $\mathrm{L}$. & -- & -- & $0.34(2.76)$ & $2.70(2.36)$ \\
\hline Xanthium strumarium L. & -- & -- & $2.07(4.58)$ & -- \\
\hline
\end{tabular}

The DCA ordination reflects that the vegetation groups obtained by TWINSPAN classification were markedly distinguishable and having a clear pattern of segregation on the ordination plane. Groups A and B (Plantago squarosa community) which are closely related to each other were separated at the right side of the DCA diagram. Group C (Plantago lagopus community) was located in the middle part, while group D (Plantago major community) was obviously separated at the left side. It is also noticed that the four vegetation groups (A-D) were clearly separated from each other, where groups A and B seemed to be closely related to each other as in groups $\mathrm{C}$ and $\mathrm{D}$ which seemed to be similar. This may be attributed to the 
similarities in the floristic structure of each pair of the identified groups as shown in figure (3).

\section{Soil characteristics}

The spatial variations in the physical and chemical characteristics of the soil samples collected from different sites in the study area are presented in table (2). The soil texture analysis revealed generally that the soil varied from loamy-sand, loamy to sandy-loamy in texture with low contents of both silt and clay fractions, where it was comparable in the three studied Plantago species.

The percentage of porosity was obviously comparable in the collected soil samples. In the soil samples of Plantago lagopus, the percentages of porosity varied from 23.20 to $49.04 \%$ with a mean value of $31.87 \%$. However, water holding capacity was obviously varied (Table 2). The chemical characteristics of the soil samples revealed that organic carbon content, electrical conductivity, and total dissolved phosphorus showed the highest significant variations between the different Plantago communities. Moreover, $\mathrm{pH}$ values, and sulfates showed significantly moderate variations between the different Plantago communities. Also, calcium carbonate content, total nitrogen, $\mathrm{Na}^{+}$and $\mathrm{K}^{+}$showed low significant variations between the different Plantago communities. On the other hand, chlorides, bicarbonates, and extractable cations $\left(\mathrm{Ca}^{2+}\right.$ and $\left.\mathrm{Mg}^{2+}\right)$ showed non-significant variations between the different Plantago communities (Table 2).

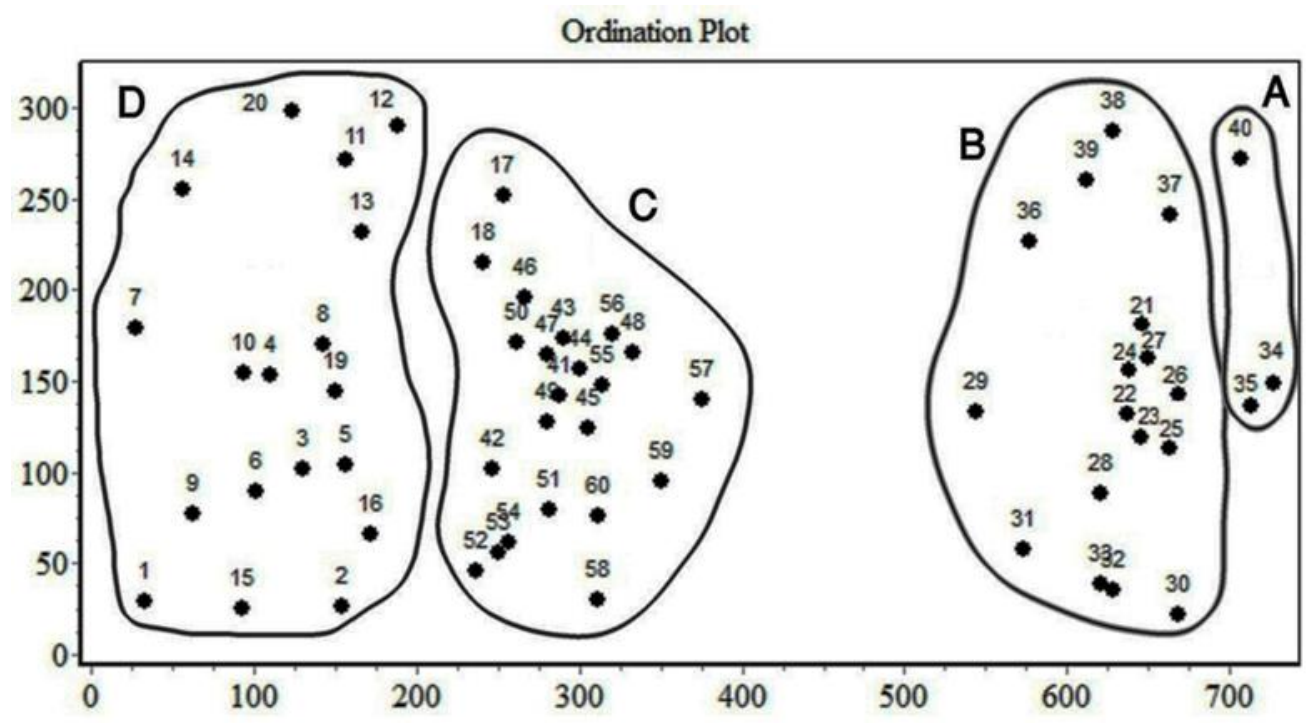

Figure (3): Detrended Correspondence Analysis (DCA) ordination diagram of 60 sampled stands within the study area.

Table (2): Maximum, minimum and mean values of the different soil variables representing the different Plantago communities.

\begin{tabular}{|c|c|c|c|c|c|c|c|c|c|c|}
\hline \multirow{2}{*}{ Soil variables } & \multicolumn{3}{|c|}{ Plantago lagopus } & \multicolumn{3}{|c|}{ Plantago major } & \multicolumn{3}{|c|}{ Plantago squarrosa } & \multirow{2}{*}{$\mathbf{L S D}_{0.05}$} \\
\hline & Max. & Min. & Mean & Max. & Min. & Mean & Max. & Min. & Mean & \\
\hline Sand\% & 99.76 & 15.40 & $82.54^{\mathrm{a}}$ & 98.44 & 18.70 & $45.35^{\mathrm{b}}$ & 98.51 & 19.60 & $72.99^{\mathrm{a}}$ & 19.24 \\
\hline Silt \% & 41.70 & 0.23 & $10.55^{\mathrm{b}}$ & 45.70 & 1.50 & $31.18^{\mathrm{a}}$ & 45.80 & 1.40 & $17.8^{\mathrm{b}}$ & 9.83 \\
\hline Clay \% & 47.60 & 0.01 & $6.91^{\mathrm{b}}$ & 36.70 & 0.01 & $23.49^{\mathrm{a}}$ & 34.60 & 0.09 & $9.21^{\mathrm{b}}$ & 9.63 \\
\hline Porosity \% & 49.04 & 23.20 & $31.87^{\mathrm{a}}$ & 42.19 & 24.60 & $33.61^{\mathrm{a}}$ & 39.68 & 21.76 & $31.13^{\mathrm{a}}$ & 3.29 \\
\hline WHC\% & 48.53 & 22.57 & $30.39^{\mathrm{c}}$ & 67.24 & 19.88 & $48.14^{\mathrm{a}}$ & 53.04 & 21.83 & $40.12^{\mathrm{b}}$ & 7.82 \\
\hline $\mathrm{CaCO}_{3} \%$ & 29.00 & 1.00 & $7.59^{\mathrm{b}}$ & 12.50 & 2.00 & $6.83^{b}$ & 19.00 & 1.25 & $11.84^{\mathrm{a}}$ & 4.03 \\
\hline $\mathrm{OC} \%$ & 2.76 & 0.18 & $1.09^{\mathrm{b}}$ & 1.86 & 0.08 & $0.87^{\mathrm{b}}$ & 3.48 & 0.16 & $2.07^{\mathrm{a}}$ & 0.42 \\
\hline pH & 9.63 & 7.71 & $8.92^{\mathrm{b}}$ & 9.49 & 8.10 & $8.89^{\mathrm{b}}$ & 10.03 & 8.70 & $9.47^{\mathrm{a}}$ & 0.34 \\
\hline $\mathbf{E C}(\mathrm{mS} / \mathrm{cm})$ & 0.44 & 0.02 & $0.12^{\mathrm{b}}$ & 1.41 & 0.01 & $0.52^{\mathrm{a}}$ & 0.32 & 0.02 & $0.11^{\mathrm{b}}$ & 0.19 \\
\hline $\mathrm{Cl}^{-} \%$ & 0.21 & 0.01 & $0.07^{\mathrm{a}}$ & 0.21 & 0.02 & $0.10^{\mathrm{a}}$ & 0.20 & 0.02 & $0.09^{\mathrm{a}}$ & 0.04 \\
\hline $\mathrm{SO}_{4}^{-2} \%$ & 0.29 & 0.01 & $0.08^{\mathrm{a}}$ & 0.08 & 0.01 & $0.04^{\mathrm{b}}$ & 0.11 & 0.01 & $0.04^{\mathrm{b}}$ & 0.03 \\
\hline $\mathrm{HCO}_{3}^{-} \%$ & 0.13 & 0.02 & $0.05^{\mathrm{a}}$ & 0.11 & 0.00 & $0.05^{\mathrm{a}}$ & 0.13 & 0.02 & $0.06^{\mathrm{a}}$ & 0.02 \\
\hline TDP (mg/100g dry soil) & 4.17 & 0.02 & $0.87^{\mathrm{b}}$ & 4.57 & 0.15 & $3.12^{\mathrm{a}}$ & 4.58 & 0.14 & $1.78^{\mathrm{b}}$ & 0.99 \\
\hline TN (mg/100g dry soil) & 9.39 & 0.01 & $1.92^{\mathrm{a}}$ & 3.67 & 0.01 & $2.35^{\mathrm{b}}$ & 3.46 & 0.01 & $0.92^{\mathrm{b}}$ & 1.24 \\
\hline $\mathrm{Na}^{+}$(mg/100g dry soil) & 96.06 & 2.57 & $24.77^{\mathrm{b}}$ & 88.01 & 10.20 & $28.67^{\mathrm{b}}$ & 93.30 & 11.60 & $47.52^{\mathrm{a}}$ & 16.10 \\
\hline $\mathrm{K}^{+}$(mg/100g dry soil) & 99.36 & 2.74 & $17.94^{\mathrm{a}}$ & 16.03 & 2.35 & $8.07^{\mathrm{b}}$ & 17.59 & 2.74 & $8.27^{\mathrm{b}}$ & 8.08 \\
\hline $\mathrm{Ca}^{+2}(\mathrm{mg} / 100 \mathrm{~g}$ dry soil $)$ & 124.25 & 3.60 & $20.73^{\mathrm{a}}$ & 35.00 & 4.40 & $18.88^{\mathrm{a}}$ & 38.80 & 4.20 & $12.27^{\mathrm{a}}$ & 10.84 \\
\hline $\operatorname{Mg}^{+2}(\mathrm{mg} / \mathbf{1 0 0 g}$ dry soil $)$ & 70.53 & 1.22 & $12.12^{\mathrm{a}}$ & 11.52 & 1.92 & $6.70^{\mathrm{a}}$ & 19.56 & 1.92 & $9.57^{\mathrm{a}}$ & 6.12 \\
\hline
\end{tabular}

\section{Vegetation-Soil Relationships}

The variation in soil variables between the vegetation groups obtained by TWINSPAN indicated that all the identified vegetation groups (A, B, C and D) showed the highest significant variation in $\mathrm{pH}$ values, where the group (A) attained the highest value (9.7) and group (D) the lowest value (8.8) (Table 3). Moreover, vegetation groups (A, B, C and D) attained moderately significant variations in the percentages of organic carbon content, electrical conductivity sulfates. The vegetation groups 
(A, B, C and D) showed low significant variations in calcium, water-holding capacity, and the total nitrogen content. On the other hand, the vegetation groups (A, B, $\mathrm{C}$ and $\mathrm{D})$ exhibited non-significant variations in the remaining soil variables as shown in table (3). Concerning the moderate significant variations, it has been noticed that the organic carbon content recorded the highest value (2.08\%) in group (A) and the lowest value $(0.96 \%)$ in group (D), while the electrical conductivity attained the highest value $(0.65 \mu \mathrm{mhos} / \mathrm{cm})$ in group (D) and the lowest value $(0.11 \%)$ in group (B). Moreover, sulfates content attained the highest value $(0.10 \%)$ in the group $(\mathrm{C})$ and the lowest value $(0.03 \%)$ in the group (A).

Table (3): Mean and standard error of the soil variables in the stands representing the vegetation groups obtained by TWINSPAN classification.

\begin{tabular}{|c|c|c|c|c|c|}
\hline \multirow[b]{2}{*}{ Soil variables } & \multicolumn{2}{|c|}{ TWINSPAN } & \multicolumn{2}{|c|}{ "Vegetation Group } & \multirow{2}{*}{$\mathbf{L S D}_{0.05}$} \\
\hline & $\mathbf{A}$ & B & $\mathbf{C}$ & D & \\
\hline Sand\% & $62.12^{\mathrm{a}} \pm 23.57$ & $71.66^{\mathrm{a}} \pm 6.91$ & $77.68^{\mathrm{a}} \pm 6.03$ & $48.40^{\mathrm{a}} \pm 8.07$ & 32.37 \\
\hline Silt \% & $21.16^{\mathrm{ab}} \pm 8.61$ & $18.56^{\mathrm{ab}} \pm 3.13$ & $12.28^{\mathrm{b}} \pm 2.86$ & $29.50^{\mathrm{a}} \pm 4.39$ & 15.39 \\
\hline Clay \% & $16.72^{\mathrm{a}} \pm 015.44$ & $9.78^{\mathrm{a}} \pm 3.46$ & $10.04^{\mathrm{a}} \pm 3.22$ & $22.11^{\mathrm{a}} \pm 3.69$ & 17.58 \\
\hline Porosity \% & $29.01^{\mathrm{a}} \pm 2.06$ & $31.47^{\mathrm{a}} \pm 2.21$ & $32.10^{\mathrm{a}} \pm 1.29$ & $33.75^{\mathrm{a}} \pm 1.18$ & 4.80 \\
\hline WHC\% & $39.78^{\mathrm{ab}} \pm 4.21$ & $39.13^{\mathrm{ab}} \pm 3.09$ & $31.11^{\mathrm{b}} \pm 1.64$ & $49.47^{\mathrm{a}} \pm 4.21$ & 12.34 \\
\hline $\mathrm{CaCO}_{3} \%$ & $8.75^{\mathrm{a}} \pm 3.03$ & $12.86^{\mathrm{a}} \pm 1.32$ & $9.58^{\mathrm{a}} \pm 1.92$ & $6.58^{\mathrm{a}} \pm 0.78$ & 6.70 \\
\hline $\mathrm{OC} \%$ & $2.08^{\mathrm{a}} \pm 0.36$ & $1.88^{\mathrm{a}} \pm 0.19$ & $1.02^{\mathrm{b}} \pm 0.15$ & $0.96^{\mathrm{b}} \pm 0.13$ & 0.70 \\
\hline pH & $9.66^{\mathrm{a}} \pm 0.13$ & $9.42^{\mathrm{a}} \pm 0.66$ & $8.83^{\mathrm{b}} \pm 0.16$ & $8.82^{\mathrm{b}} \pm 0.08$ & 0.44 \\
\hline $\mathrm{EC}(\mathrm{mS} / \mathrm{cm})$ & $0.16^{\mathrm{b}} \pm .08$ & $0.11^{\mathrm{b}} \pm 0.02$ & $0.14^{\mathrm{b}} \pm 0.03$ & $0.65^{\mathrm{a}} \pm 0.12$ & 0.31 \\
\hline $\mathrm{Cl}^{-} \%$ & $0.07^{\mathrm{a}} \pm 0.02$ & $0.08^{\mathrm{a}} \pm 0.01$ & $0.10^{\mathrm{a}} \pm 0.01$ & $0.11^{\mathrm{a}} \pm 0.01$ & 0.06 \\
\hline $\mathrm{SO}_{4}^{-2} \%$ & $0.03^{\mathrm{b}} \pm 0.01$ & $0.04^{\mathrm{b}} \pm 0.01$ & $0.10^{\mathrm{a}} \pm 0.02$ & $0.05^{\mathrm{b}} \pm 0.01$ & 0.04 \\
\hline $\mathrm{HCO}_{3}^{-} \%$ & $0.05^{\mathrm{b}} \pm 0.01$ & $0.05^{\mathrm{ab}} \pm 0.01$ & $0.08^{\mathrm{a}} \pm 0.32$ & $0.06^{\mathrm{ab}} \pm 0.01$ & 0.03 \\
\hline TDP (mg/100g dry soil) & $1.86^{\mathrm{ab}} \pm 0.31$ & $1.05^{\mathrm{b}} \pm 0.30$ & $2.95^{\mathrm{a}} \pm 0.44$ & $31.90^{\mathrm{a}} \pm 5.48$ & 1.56 \\
\hline TN (mg/100g dry soil) & $0.98^{\mathrm{b}} \pm 0.35$ & $14.01^{\mathrm{a}} \pm 5.32$ & $2.21^{\mathrm{b}} \pm 0.37$ & $8.25^{\mathrm{a}} \pm 1.04$ & 10.46 \\
\hline $\mathrm{Na}^{+}$(mg/100g dry soil) & $40.31^{\mathrm{a}} \pm 6.03$ & $35.06^{\mathrm{a}} \pm 5.88$ & $31.90^{\mathrm{a}} \pm 5.48$ & $21.2^{\mathrm{ab}} \pm 2.51$ & 26.17 \\
\hline $\mathrm{K}^{+}(\mathrm{mg} / 100 \mathrm{~g}$ dry soil $)$ & $7.69^{\mathrm{a}} \pm 0.96$ & $22.17^{\mathrm{a}} \pm 5.30$ & $8.25^{\mathrm{a}} \pm 1.04$ & $7.57^{\mathrm{a}} \pm 0.82$ & 14.56 \\
\hline $\mathrm{Ca}^{+2}$ (mg/100g dry soil) & $13.02^{\mathrm{b}} \pm 1.83$ & $33.78^{\mathrm{a}} \pm 5.65$ & $21.2^{\mathrm{ab}} \pm 2.51$ & $9.45^{\mathrm{a}} \pm 1.53$ & 18.58 \\
\hline $\mathbf{M g}^{+2}$ (mg/100g dry soil) & $8.28^{\mathrm{a}} \pm 1.93$ & $9.67^{\mathrm{a}} \pm 1.30$ & $18.31^{\mathrm{a}} \pm 3.29$ & $7.57^{\mathrm{a}} \pm 0.82$ & 10.79 \\
\hline
\end{tabular}

On the other hand, concerning the low significant variations, it has been found that water-holding capacity showed the highest value $(49.47 \%)$ in the group (D) and the lowest value $(31.11 \%)$ in the group (C). While total nitrogen content exhibited the highest value (14.01 $\mathrm{mg} / 100 \mathrm{~g}$ dry soil) in the group (B) and the lowest value $(0.98 \mathrm{mg} / 100 \mathrm{~g}$ dry soil) in the group (A). Calcium cation attained the highest value $(33.78 \mathrm{mg} / 100 \mathrm{~g}$ dry soil) in the group (B) and the lowest value (9.45 $\mathrm{mg} / 100 \mathrm{~g}$ dry soil) in the group (D).

The results of plant-soil variables Pearson moment correlation are shown in table (4). The sand showed negatively high significant correlations with Sonchus oleraceus. While, it attained a positively low significant correlation with two plant species namely, Emex spinosa and Plantago lagopus. Silt exhibited a positively high significance with Sonchus oleraceus, while it attained a negatively moderate correlation with Emex spinosa, but a low significant correlation with Plantago lagopus. Clay showed a positively moderate correlation with Sonchus oleraceus, and a positively low correlation was attained with Elymus farctus. Furthermore, a negatively low correlation was attained with Emex spinosa. Water-holding capacity also attained a positively high significant correlation with Sonchus oleraceus. It showed negatively moderate correlations with Emex spinosa and Plantago lagopus as well as a low significant correlation with Lolium multiflorum.

Calcium carbonate exhibited a low positively significant correlation with Plantago squarrosa, and a negatively moderate significant correlation with Lolium multiflorum. Organic carbon attained positively high significant correlations with Erodium laciniatum, Rum- ex pictus, and Plantago squarrosa. It showed also a moderately positive significant correlation with Emex spinosa and a negative correlation with Plantago major. Although it exhibited low positive significant correlations with Aegilops bicornis and Stipagrostis lanata, it attained negative correlations with Malva parviflora and Rumex dentatus. The $\mathrm{pH}$ showed a positively moderate correlation with Erodium laciniatum as well as low significant correlations with Plantago squarrosa and Emex spinosa. It exhibited also a negatively low significant correlation with Cynodon dactylon. Electrical conductivity exhibited a high positively significant correlation with Rumex dentatus a moderate significant correlation with Sonchus oleraceus and a positively low significant correlation with Malva parviflora.

Chlorides exhibited a positively moderate significant correlation with Rumex dentatus and a positively low significant correlation with Malva parviflora. Sulphates exhibited a positively low significant correlation with Cynodon dactylon. Bicarbonates showed a positively high significant correlation with Plantago lagopus. Total dissolved phosphorus exhibited a high positively significant correlation with Sonchus oleraceus. As well as it showed a negatively moderate correlation with Emex spinosa and a low correlation with Plantago lagopus was attained. Total nitrogen attained a low positively significant correlation with Cynodon dactylon.

Sodium has a positively moderate correlation with Erodium laciniatum. While, low positively significant correlations with Echinops spinosus and Rumex pictus, as well as it negatively correlated with Emex spinosa. On the other hand, porosity, calcium, and magnesium didn't exhibit any correlation with any plant species. 
Table (4): Pearson-moment correlation (r) between the the importance values (based on density and cover) of the dominant and important plant species and soil variables.

\begin{tabular}{|c|c|c|c|c|c|c|c|c|c|c|c|c|c|c|c|c|}
\hline $\begin{array}{l}\text { Plant } \\
\text { species }\end{array}$ & 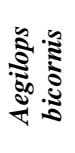 & 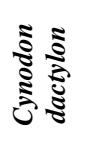 & 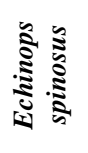 & 言总 & 悹言 & 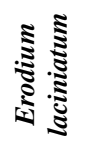 & 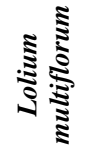 & 胥 & 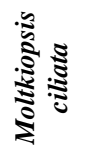 & 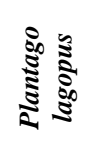 & 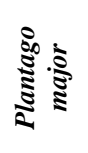 & 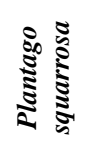 & 气 & 气 & 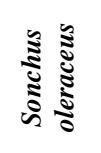 & 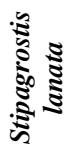 \\
\hline Sand & 0.06 & 0.13 & 0.15 & -0.21 & $0.32^{*}$ & 0.14 & 0.11 & -0.23 & 0.1 & $0.26^{*}$ & -0.15 & 0.02 & -0.18 & -0.01 & $-0.44^{* * *}$ & -0.13 \\
\hline Silt & -0.03 & -0.15 & -0.14 & 0.14 & $-0.35^{* *}$ & -0.1 & -0.16 & 0.24 & -0.06 & $-0.28^{*}$ & 0.14 & 0.02 & 0.18 & 0.05 & $0.45^{* * *}$ & 0.08 \\
\hline Clay & -0.08 & -0.1 & -0.17 & $0.28^{*}$ & $-0.27^{*}$ & -0.18 & -0.07 & 0.23 & -0.13 & -0.24 & 0.16 & -0.05 & 0.18 & -0.05 & $0.41^{* *}$ & 0.18 \\
\hline Porosity & -0.18 & 0.06 & 0.02 & -0.15 & -0.12 & -0.07 & -0.19 & 0 & -0.05 & -0.03 & 0.02 & -0.13 & 0.01 & -0.04 & 0.24 & -0.18 \\
\hline WHC & -0.06 & -0.11 & 0.11 & 0.09 & $-0.36^{* * *}$ & 0 & $-0.26^{*}$ & 0.14 & -0.05 & $-0.35^{* *}$ & 0.13 & 0.03 & 0.18 & 0.15 & $0.44^{* * *}$ & 0.06 \\
\hline $\mathrm{CaCO}_{3}$ & 0 & 0.13 & 0.17 & -0.12 & -0.22 & 0.19 & $-0.40^{* * *}$ & -0.1 & 0.08 & -0.15 & -0.23 & $0.29^{*}$ & -0.23 & 0.2 & -0.03 & -0.06 \\
\hline OC & $0.26^{*}$ & -0.25 & $0.41^{* *}$ & 0.22 & -0.17 & $0.42^{* * * *}$ & -0.03 & $-0.27^{*}$ & 0.08 & -0.1 & $-0.41^{* *}$ & $0.48^{* * *}$ & $-0.31^{*}$ & $0.48^{* * * *}$ & -0.17 & $0.26^{*}$ \\
\hline pH & 0.13 & $-0.27^{*}$ & $0.32^{*}$ & 0.07 & -0.02 & $0.37^{7^{* *}}$ & 0.23 & -0.19 & 0.21 & 0 & -0.16 & $0.32^{*}$ & -0.21 & 0.2 & -0.05 & 0.18 \\
\hline EC & -0.14 & 0.05 & -0.14 & 0.03 & -0.22 & -0.17 & -0.22 & $0.31^{*}$ & -0.09 & -0.19 & 0.18 & -0.23 & $0.41^{* * *}$ & -0.14 & $0.34^{* *}$ & -0.02 \\
\hline $\mathrm{Cl}^{-}$ & 0 & -0.04 & 0.16 & -0.12 & -0.08 & 0.06 & -0.08 & $0.33^{*}$ & 0 & -0.17 & 0.04 & -0.06 & $0.37^{* *}$ & 0.19 & -0.07 & -0.1 \\
\hline $\mathrm{SO}_{4}^{-2}$ & -0.08 & $0.28^{*}$ & -0.05 & -0.1 & 0.21 & -0.11 & -0.14 & 0.05 & -0.06 & 0.15 & -0.2 & -0.2 & 0.01 & -0.04 & -0.14 & -0.12 \\
\hline $\mathrm{HCO}_{3}^{-}$ & -0.05 & -0.1 & -0.04 & -0.02 & -0.04 & -0.05 & 0.19 & -0.05 & -0.02 & $0.49^{* * * *}$ & -0.08 & -0.08 & 0.09 & -0.05 & 0.01 & -0.03 \\
\hline TDP & -0.02 & -0.17 & -0.12 & 0.13 & $-0.37^{* *}$ & -0.08 & -0.14 & 0.21 & -0.05 & $-0.29^{*}$ & 0.15 & 0.03 & 0.19 & 0.06 & $0.45^{* * *}$ & 0.08 \\
\hline TN & -0.11 & $0.32^{*}$ & -0.12 & -0.01 & 0.12 & -0.14 & -0.13 & -0.03 & -0.07 & 0.11 & -0.13 & -0.17 & -0.12 & -0.13 & -0.06 & -0.04 \\
\hline $\mathrm{Na}^{+}$ & 0.11 & -0.05 & $0.32^{*}$ & -0.06 & $-0.27^{*}$ & $0.38^{8^{* *}}$ & -0.05 & 0.07 & 0.17 & -0.17 & -0.1 & 0.24 & -0.16 & $0.26^{*}$ & -0.04 & 0.04 \\
\hline $\mathbf{K}^{+}$ & -0.1 & -0.04 & -0.04 & -0.07 & 0.01 & -0.09 & 0.08 & 0.28 & -0.04 & $0.37^{* *}$ & -0.2 & -0.18 & -0.06 & -0.07 & -0.09 & -0.08 \\
\hline $\mathrm{Ca}^{+2}$ & -0.06 & 0.17 & -0.07 & -0.1 & -0.15 & -0.12 & -0.15 & 0.51 & -0.08 & -0.02 & 0.01 & -0.19 & 0.2 & -0.04 & -0.07 & -0.13 \\
\hline $\mathrm{Mg}^{+2}$ & -0.04 & -0.09 & -0.02 & -0.07 & 0.04 & 0.06 & -0.03 & 0.4 & 0.01 & 0.01 & -0.18 & 0.02 & -0.05 & 0.06 & -0.16 & -0.05 \\
\hline
\end{tabular}

It is noticed that as shown in figure (4) organic carbon, electrical conductivity, sand fraction and $\mathrm{pH}$ value showed highly significant correlations with the first and second axes. While, calcium carbonate, sodium, potassium and calcium cations as well as water-holding capacity, total dissolved phosphorus, silt and clay fractions exhibited a moderate significant correlation. On the other hand, total nitrogen, chlorides, sulfates, bicarbonates, porosity, and magnesium showed low significant correlations with the first and second axes of the CCA diagram. In the right side of CCA diagram, Plantago squarrosa (dominant plant species in groups A \& B), Moltkiopsis ciliata, Stipagrostis lanata (important species in group A), Emex spinosa, Erodium laciniatum, Aegilops bicornis (important species in group B) and Rumex pictus were obviously controlled by many soil variables such as calcium carbonate, sodium, organic carbon, pH value, sand fraction and magnesium (Fig. 4).

In the upper left side Plantago lagopus (dominant plant species of group C),

Cynodon dactylon (important species in group D) Lolium multiflorum and Emex spinosa (important species in group C) were clearly affected by bicarbonates, potassium, sulfates and total nitrogen (Fig. 4). In the lower left side, Plantago major (dominant plant species of group D), Rumex dentatus, Sonchus oleraceus and Cynodon dactylon (important species in group D) showed close relationships with electrical conductivity, water-holding capacity, total dissolved phosphorus, silt, clay and porosity (Fig. 4).

\section{Diversity measurements of vegetation groups}

It is obvious that Plantago major community (group D) attained the highest value 3.13 of the Shannon-Wiener $\left(\mathrm{H}^{\prime}\right)$, followed by Plantago lagopus community (group C) which attained the value of 2.88 . While, Plantago squarrosa community (Groups A and B) showed the lowest values 1.81 and 2.44, respectively (Fig. a).

The vegetation groups obtained from TWINSPAN classification demonstrated differences in Shannon-evenness diversity index (E) as shown in figure (5b). 


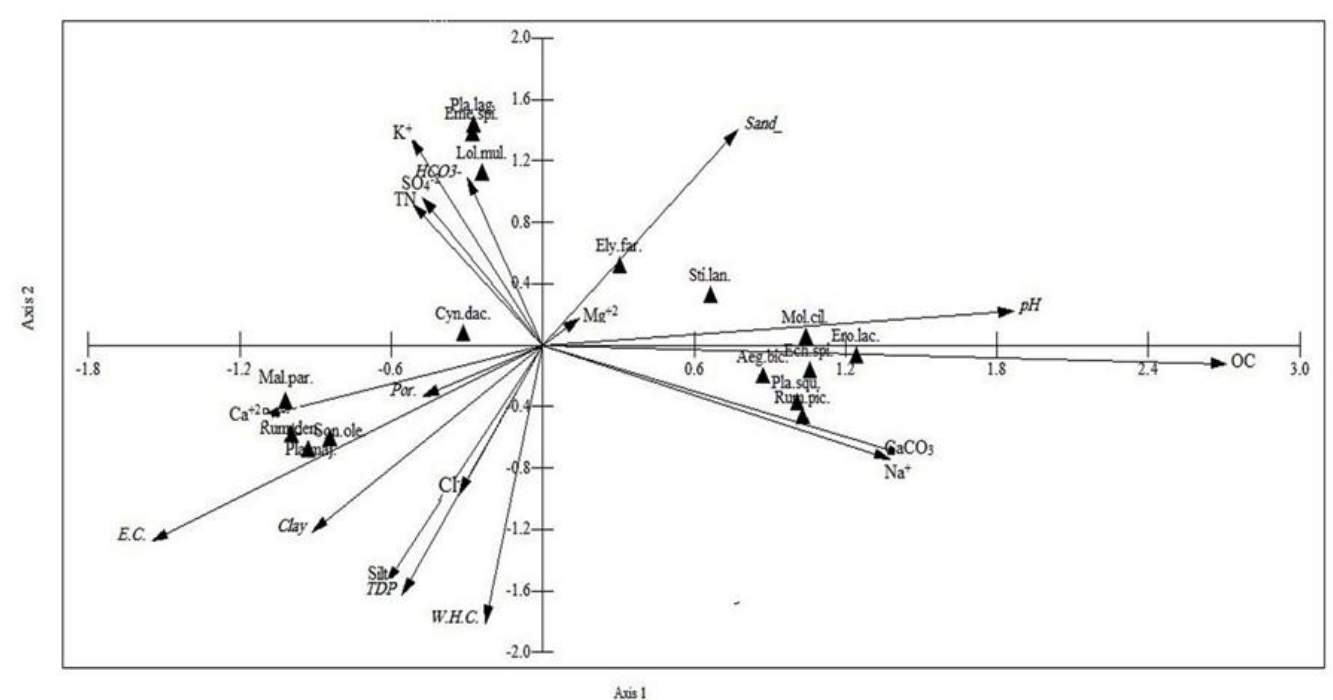

Figure (4): Canonical Correspondence Analysis (CCA) of the plant species along the environmental gradients (arrows).

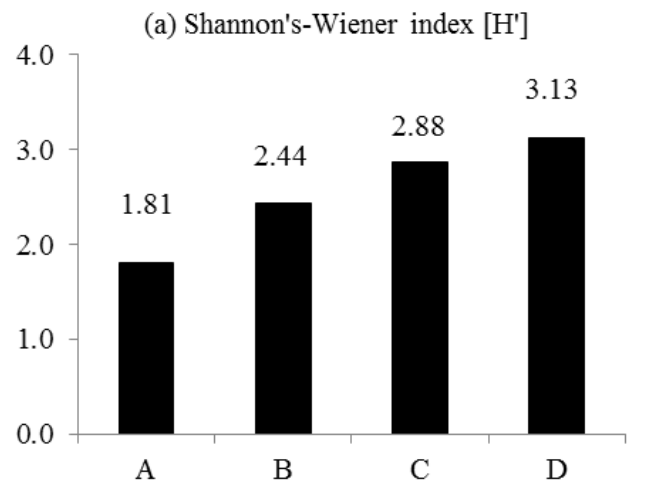

(b) Shanon's-Evennes index [E]

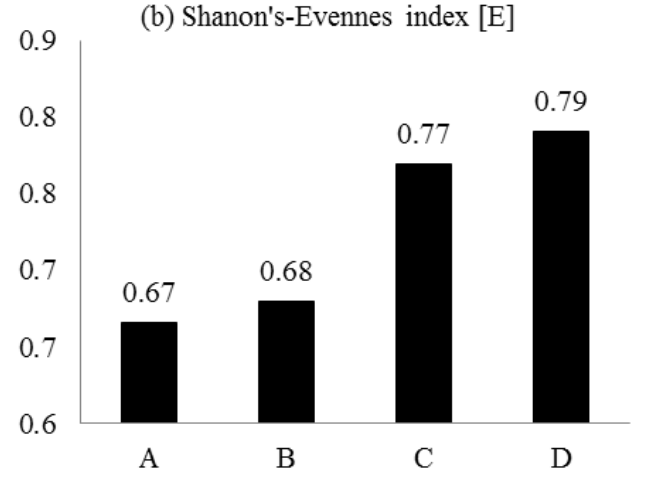

(c) Simpson's diversity index (D)

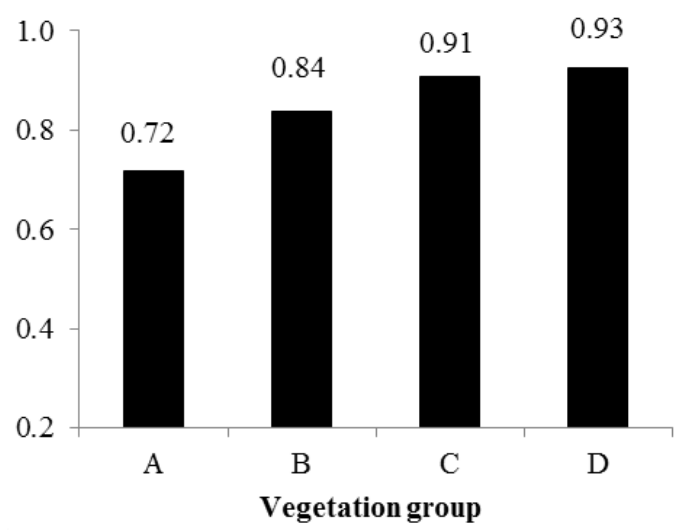

Figure (5): Diversity indices of the four vegetation groups derived from the TWINSPAN classification.
Plantago major community (group D) attained also the highest values of Shannon-evenness diversity index (0.79) followed by Plantago lagopus community (group C) which attained the value of 0.77 , while Plantago squarrosa community (Groups A and B) attained the lowest values ( 0.68 and 0.67 , respectively) (Fig. 5b).

The vegetation groups also demonstrated differences in the Simpson's diversity index (D) as shown in figure (5c). It is clear that; Plantago major community (group D) attained the highest value 0.93 of Simpson's diversity index, followed by Plantago lagopus community (group C) which attained the value of 0.91 , while, Plantago squarrosa community (Groups A and B) attained the lowest values 0.72 and 0.84 , respectively of Simpson's diversity index.

\section{DISCUSSION}

Plantaginaceae is a cosmopolitan family which comprises some worldwide weeds mainly annual or perennial herbs stemless or short-stemmed. Approximately it has 3 genera most of its taxa are in Plantago (Huisinga and Ayers, 1999). Plantago species have been found in temperate and in tropical zones, including the varied ecological systems required by the plant to adapt both phenotypically and physiologically (Kuiper, 1992; Van Delden et al., 1992). Many types of research in diverse areas have been carried out on Plantago species. Changes in the concentrations of bioactive compounds in plantain species occurred under various natural climatic conditions (Fons et al., 2008).

Modern synecological studies have preferred more objective methodology for use at a local and sometimes regional scale. These have sought to reduce the complexity of a set of field data either by classification and/or ordination based on floristic data. The results of vegetation analysis have then been related to environ-mental data. Alternatively, vegetation-habitat relationships have been derived from a single analysis of combined floristic and environmental variables (ter Braak, 1987).

The application of TWINSPAN classification based on the importance values of 105 plant species recorded 
in 60 sampled stands representing different habitat types of the study area, led to the recognition of four vegetation groups. Each group comprises a number of sampled stands which are similar in terms of vegetation and characterized by dominant and/or codominant species as well as by a number of the indicator and/or preferential species.

Group (A) comprises 3 stands dominated by Plantago squarrosa which has the highest importance value of this group. The other important and indicator species which attained relatively high importance values in this group were Moltkiopsis ciliata, Stipagrostis lanata and Echinops spinosus. Group (B) includes 17 stands dominated also by Plantago squarrosa. The other important and indicator species which attained relatively high importance values in this group were Erodium laciniatum, Aegilops bicornis, Echinops spinosus and Bromus catharactius. Group C comprises 21 stands dominated by Plantago lagopus. Cynodon dactylon, Lolium multiflorum and Emex spinosa were the important species in this group. The indicator species in this group include Chenopodium murale, Raphanus raphainstrum and Urospermum picroides. Group D comprises 19 stands dominated by Plantago major. The other important species were Rumex dentatus, Cynodon dactylon, Sonchus oleraceus, Malva parviflora and Lotus glaber.

The associations of the vegetation analysis recognized in the cultivated land habitat in the present study may be similar to the associations described by Shaltout et al. (1992) on the weed communities of the common crops in the Nile Delta, Shalaby (1995) on plant life at Kafr El- Sheikh Province, Sheded and Turki (2000) on the weed flora of field crops and orchards in south Nile delta, El-Halawany et al. (2002) on the weed communities of the principal crops in Damietta Governorate, Mashaly (2003) on the weed flora of the main crops in Kafr El-Sheikh Governoate, Mashaly and Awad (2003) on the weed flora of orchards in the Nile delta region, Baraka and Al-Sodany (2003) on the habitat and plant life in Sharkia Governorate in Nile Delta, El-Halawany et al. (2010) on the habitat and plant life in El-Dakahlia Governorate, and Mashaly et al. (2013) on vegetationsoil relationship in the cultivated land in El-Behira Governorate. Generally, the vegetation groups identified in the present study were more or less similar to most of the previously mentioned studies.

The application of DCA ordination in the sampled stands indicated that the vegetation groups derived by TWINSPAN classification are more or less distingueishable and having a clear pattern of the distinction between different vegetation groups on the ordination planes. All the vegetation groups in the present study are located on the positive side of the first and second ordination axes. Groups (A) and (B) (Plantago squarrosa community) were separated at the right side of the DCA diagram, Group (C) (Plantago lagopus community) was in the middle part of DCA diagram, while group (D) (Plantago major community) was obviously separated at the left side. It is also noticed that the four vegetation groups (A-D) were clearly separated from each other, where groups (A) and (B) seemed to be closely related to each other as in groups (C) and (D) which seemed to be similar. This may be attributed to the similarities in the floristic structure of each pair of the identified groups.

Plantago lagopus community (group C) attained a loamy-sand soil. It had values more than $30 \%$ of waterholding capacity; on the other hand the highest values of potassium, total nitrogen content, calcium, and magnesium. It exhibited moderate values of electrical conductivity, sulfates and organic carbon. Plantago major community (group D) attained a loamy textured soil with the highest values of water-holding capacity, organic carbon, and electrical conductivity. Plantago squarrosa community (groups $\mathrm{A}$ and $\mathrm{B}$ ) attained a sandy-loamy textured soil with highest values of total dissolved phosphorus. All studied Plantago communities indicated slightly alkaline soil reactions with values more than $30 \%$ of porosity. This agrees more or less with the studies of Omar (2006) on the plant life in the northern Nile Delta and Abd El-Gawad (2008) on the ecology of some non-conventional forage weeds in the same region.

Plantago lagopus community showed positively significant correlations with sand fraction, and potassium. It attained negative correlations with silt fraction and total dissolved phosphorus. These results agree, more or less, with other studies such as Abd El-Ghani et al. (2014a) and Salama et al. (2013).

Plantago major community attained negatively significant correlations with organic carbon and bicarbonates, while Plantago squarrosa community showed positively significant correlations with organic carbon, calcium carbonates, and $\mathrm{pH}$. This was also reported in other studies (e.g. Abd El-Ghani et al. 2014a and b).

Echinops spinosus, Emex spinosa, Erodium laciniatum, Malva parviflora Rumex dentatus and Sonchus oleraceus associations correlated significantly with organic carbon, this was also reported by Abd El-Gawad (2014). Echinops spinosus correlated significantly with pH, and sodium; Emex spinosa correlated with soil fractions, sodium, and water-holding capacity; while Erodium laciniatum correlated significantly with $\mathrm{pH}$, total dissolved phosphorus, and sodium. On the other hand, Malva parviflora and Rumex dentatus were significantly correlated with electrical conductivity, chlorides, and sulfates. Sonchus oleraceus correlated with soil fractions, water-holding capacity, electrical conductivity, sulfates and total dissolved phosphorus. This agrees more or less with the study of Abu-Ziada et al. (2008).

In the present phytosociological study, the application of CCA bi-plot between the position of vegetation groups on the ordination planes and soil variables of their stands indicated that, the most important soil factors that controlling the distribution and abundance of vegetation groups were organic carbon, electrical conductivity, sand fraction and $\mathrm{pH}$ value which showed relatively high significant correlations with the first and second axes. In addition, calcium carbonate, sodium, potassium and calcium cations, water-holding capacity, total dissolved phosphorus, silt and clay fractions exhibited a moderate significant correlation. On the 
other hand, total nitrogen, chlorides, sulfates, bicarbonates, porosity, and magnesium showed relatively low significant correlations with the first and second axes of the CCA diagram. In the right side of CCA diagram, Plantago squarrosa (dominant plant species of groups A and B), Moltkiopsis ciliata, Stipagrostis lanata (important species in group A), Emex spinosa, Erodium laciniatum, Aegilops bicornis (important species in group B) and Rumex pictures were obviously affected with many soil variables such as calcium carbonate, sodium, organic carbon, $\mathrm{pH}$ value, sand and magnesium.

In the upper left side Plantago lagopus (dominant plant species of group C), Cynodon dactylon (important species in group D) Lolium multiflorum and Emex spinosa (important species in group C) were clearly affected by bicarbonates, potassium, sulfates and total nitrogen. In the lower left side, Plantago major (dominant plant species of group D), Rumex dentatus, Sonchus oleraceus and Cynodon dactylon (important species in group D) showed a close relationship with electrical conductivity, water-holding capacity, total dissolved phosphorus, silt, clay, and porosity. These results agree, more or less, with those investigated by Shehata and El-Fahar (2000), Mashaly and Awad (2003), Galal and Fawzy (2007), Mashaly et al. (2008), Maswada (2009), Mashaly et al. (2009) and Abd ElGhani et al. (2014a).

The variations in species richness and evenness among the different habitat types may be attributed to the difference in soil characteristics, substrate discontinuities and the allelopathic effect of one or more plant species depending on their relative dominance among other associated species (EL-Khatib et al., 2004; Hegazy et al., 1994; James et al., 2006). This is in accordance with the findings of Mellinger and McNaughton (1975) that provide evidence that a high level of species diversity would be brought about by a local differentiation in soil properties around individual plants, since heterogeneity of environments allows satisfaction of the requirements of many species within a community (Whittaker and Levin, 1977). Species diversity increases as the number of species per sample increases and as the abundance of species within a sample become even (Pielou, 1969). Consequently, Plantago major community (vegetation group D) was more diverse than those of the other groups.

\section{REFERENCES}

ABD EL-GAWAD, A.M. 2008. Ecological studies on some non-conventional forage weeds in Nile delta, Egypt. M.Sc. Thesis, Mansoura University

ABD EL-GAWAD, A.M. 2014. Ecology and allelopathic control of Brassica tournefortii in reclaimed areas of the Nile delta, Egypt. Turkish Journal of Botany, 38 (2): 347-357.

ABD EL-GHANI, M., R.S. HAMDY AND A. HAMED 2014a. Aspects of vegetation and soil relationships around a thalassohaline lakes of Wadi El-Natrun, Western Desert, Egypt. Journal of Biology and Earth Sciences, 4: 21-35.
ABD EL-GHANI, M., A. SOLIMAN AND R. ABD EL-FATTAH 2014b. Spatial distribution and soil characteristics of the vegetation associated with common succulent plants in Egypt. Turkish Journal of Botany, 38 (3): 550-565.

ABD EL-GHANI, M.M., AND A.M. AMER. 1990.

Studies on Weed Assemblages in Croplands, Egypt. I. Broad Bean (faba bean) Fields. Egyptian Journal of Botany, 33 (1): 15-30.

ABD RAZIK, B.M., H.A. HASAN AND M.K. MURTADHA 2012. The study of antibacterial activity of Plantago major and Ceratonia siliqua. The Iraqi Postgraduate Medical Journal, 11 (1): 130-135.

ABU ZIADA, M.E., I.A. MASHALY AND M. TORKY 2008. Ecological studies on the aquatic vegetation in north east Nile Delta, Egypt. International Journal of Botany, 4 (2): 151-163.

AKOBUNDU, I.O. 1991. Weeds in human affairs in sub-Saharan Africa: Implications for sustainable food production. Weed Technology, 5: 680-690.

ALLEN, S.E., H.M. GRIMSHAW, J.A. PARKINSON, C. QUARMBY, AND J.D. ROBERTS. 1974. Chemical Analysis of Ecological Materials. Osney Blackwell Scientific Publ., Oxford, London.

ANONYMOUS. 1977. Annual environmental report. General directorate of meteorology. Ministry of Defense and Aviations Part I. Surface climatologically report, Kingdom of Saudi Arabia. pp. 35-90.

APHA. 1998. Standard Methods for the Examination of Water and Waste Water. 19th ed ed. American Public Health Association, American Water Work Association, Water Pollution Control Federation, Washington, D.C.

BARAKA, D.M., AND Y.M. AL-SODANY. 2003. Habitat and plant life in Sharkia Governorate, Nile delta, Egypt. Taeckholmia, 23 (1): 1-13.

BERKOWITZ, A.R. 1988. Competition for resources in weed-crop mixtures. In: Altieri MA, Liebman M (eds) Weed Management in Agroecosystems: Ecological Approaches. CRC Press, Boca Raton, Florida, USA, pp 89-120.

BOOTH, B.D., S.D. MURPHY AND C.J. SWANTON. 2003. Ecology of weeds. In: Booth BD, Murphy SD, Swanton CJ (eds) Weed Ecology in Natural and Agricultural Systems. CABI Publishing, UK, pp 114

BOULOS, L. 1999-2005. Flora of Egypt. Vols. 1-4. Al Hadara Publishing, Cairo, Egypt.

BOULOS, L. 2009. Flora of Egypt checklist, Revised annotated edition. Al Hadara Publishing, Cairo, Egypt.

CANFIELD, R. 1941. Application of the line interception method in sampling range vegetation. Journal of Forestry, 39: 288-394.

CHIKOYE, D., S.F. WEISE AND C.J. SWANTON. 1995. Influence of common ragweed (Ambrosia artemisiifolia) time of emergence and density on white bean (Phaseolus vulgaris). Weed Science, 43: 375-380.

EL-HALAWANY, E.F., I.A. MASHALY, M.E. ABUZIADA, AND M. ABD EL-AAL. 2010. Habitat and plant life in El-Dakahlyia Governorate, Egypt. 
Journal of Environmental Science, Mansoura University, 39 (1): 83-108.

EL-HALAWANY, E.F., I.A. MASHALY AND G.OMAR. 2002. On the ecology of weed communities of the principal crops in Damietta area, Egypt. Journal of Environmental Sciences, Mansoura University, 33: 95-118.

EL-KHATIB, A.A., A.K. HEGAZY AND H.K. GALAL. 2004. Allelopathy in the rhizosphere and amended soil of Chenopodium murale L. Weed Biology and Management, 4 (1): 35-42.

FONS, F., A. GARGADENNEC AND S. RAPIOR 2008. Culture of Plantago species as bioactive components resources: a 20-year review and recent applications. Acta Botanica Gallica, 155 (2): 277300.

FUHRER, G.J. 1999. The Quality of Our Nation's Waters: Nutrients and Pesticides. US Department of Interior and US Geological Survey DIANE Publishing, Washington, DC.

GALAL, T.M., AND M. FAWZY. 2007. Sand dune vegetation in the coast of Nile delta, Egypt Global Journal of Environmental Research, 2: 74-85.

GAUCH, H.G., AND R.H. WHITTAKER. 1981. Hierarchical Classification of Community Data. Journal of Ecology, 69: 537-557.

GHDIFAN, A., G. IBRAHIM, AND A. BASHEER. 2011. Survey of insect species associated with the perennial weed, Plantago spp. in Damascus region, Syria. Egyptian Journal of Biological Pest Control, 21 (1): 89-96.

GOODALL, D.W. 1954. Objective methods for the classification of vegetation. III. An essay in the use of factor analysis. Australian Journal of Botany, 2 (3): 304-324.

HARLAN, J.R. 1992. Crops and Man. 2nd ed. American Society of Agronomy, Madison, USA.

HARPUT, U.S., Y. GENC AND I. SARACOGLU. 2012. Cytotoxic and antioxidative activities of Plantago lagopus L. and characterization of its bioactive compounds. Food and Chemical Toxicology, 50 (5): 1554-1559.

HEGAZY, A.K., M.I. SOLIMAN, AND I.A. MASHALY. 1994. Perspectives on the biology of Heliotropium curassavicum in the deltaic Mediterranean coast of Egypt. Arab Gulf Journal of Scientific Research, 12: 525-545.

HENDERSON, P.A., AND R.M.H. SEABY. 1999. Community Analysis Package (CAP) version 1.2. Pisces Conservation Ltd. IRC House, UK.

HILL, M.O. 1979. DECORANA-a FORTRAN Program for Detrended Correspondence Analysis and Reciprocal Averaging. Section of Ecology and Systematic, Cornell University, Ithaca, New York.

HOLZNER, W. 1982. Concepts, categories and characteristics of weeds. In: Holzner W, Numata N (eds) Biology and Ecology of Weeds. Dr W. Junk Publishers, The Hague, The Netherlands, pp 3-20.

HUISINGA, K.D., AND T.J. AYERS. 1999. Vascular plants of Arizona: Plantaginaceae plantain family. Journal of the Arizona-Nevada Academy of Science, 32 (1): 62-76.
JACKSON, M.L. 1962. Soil Chemical Analysis. Constable and Co. LTD, London.

JAMES, J.J., M.A. CAIRD, R.E. DRENOVSKY AND R.L. SHELEY. 2006. Influence of resource pulses and perennial neighbors on the establishment of an invasive annual grass in the Mojave desert. Journal of Arid Environments, 67 (3): 528-534.

KUIPER, D. 1992. Ecophysiology of Plantago species. Phenotypic plasticity in Plantago species. In: Kuiper PJC, Bos M (eds) Plantago: A Multidisciplinary Study, vol 89. Springer, Verlag, Paris, pp 169-183

LARSON, S.J., R.J. GILLIOM, AND P.D. CAPEL. 1999. Pesticides in streams of the United States: initial results from the national water-quality assessment program vol 98. US Department of the Interior, US Geological Survey, Sacramento, California

MAGURRAN, A.E. 1988. Ecological Diversity and its Measurement. Springer Netherland, Princeton, New Jersey.

MASHALY, I.A. 2003. Phytosociological study on the weed flora of crop lands in Kafr El-Sheikh Governorate, Egypt. El-Minia Science Bulletin, 14 (2): 127-153.

MASHALY, I.A., AND E.R. AWAD. 2003. Weed flora of orchards in the Nile Delta, Egypt: Floristic features. Asian Journal Plant Science, 2: 314-324.

MASHALY, I.A., E.E. EL-HABASHY, E.F. ELHALAWANY, AND G. OMAR. 2008. Habitats and Plant communities in the Nile delta of Egypt. Ideltaic Mediterranean coastal habitat. Pakistan Journal of Biological Sciences, 11 (22): 2532-2544.

MASHALY, I.A., E.E. EL-HABASHY, E.F. ELHALAWANY, AND G. OMAR. 2009. Habitats and plant communities in the Nile delta of Egypt. IIIrrigation and drainage canal bank habitat. Pakistan Journal of Biological Sciences, 12 (12): 885-895.

MASHALY, I.A., E.F. EL-HALAWANY, AND N.A. ABD EL-HADY. 2011. Weed vegetation-soil relationship in the deltaic Mediterranean coast of Egypt. Journal of Environmental Sciences, Mansoura University, 40 (4): 501-519.

MASHALY, I.A., E.F. EL-HALAWANY, AND A.M. ABD EL-GAWAD. 2011. Ecology of weeds and invasive plant species in newly reclaimed areas in Nile delta, Egypt. Journal of Environmental Sciences, Mansoura University, 40 (1): 69-90.

MASHALY, I.A., E.F. EL-HALAWANY, M.E. ABU ZIADA, AND M.L. ABD EL-AAL. 2013. Vegetation - soil relationship in the cultivated land habitat in El-Behira Governorate, Egypt. Journal of Environmental Sciences, Mansoura University, 42 (4): 607-623.

MASWADA, H.F. 2009. Ecological and physiological studies on some geophytes in the Mediterranean coastal region of Kafr El-Sheikh Governorate, Egypt. Ph.D. Thesis, Tanta University

MELLINGER, M.V., AND S.J. MCNAUGHTON. 1975. Structure and function of successional vascular plant communities in central New York. Ecological Monographs, 45: 161-182.

MOHSENZADEH, S., V. NAZERI AND S.M. MIRTADZADINI. 2008. Chromosome numbers of 
fifteen species of Plantago L.(Plantaginaceae) from Iran. Iranian Journal of Botany, 14 (1): 47-53.

MORTENSEN, D.A., J.A. DIELEMAN AND G.A. JOHNSON. 1998. Weed spatial variation and weed management. In: Hatfield JL, Buhler DD, Stewart BA (eds) Integrated Weed and Soil Management. MI: Ann Arbor Press, Chelsea, London, pp 293-309

OMAR, G. 2006. Plant life of the different habitats in the north Nile Delta of Egypt: Ecology and fodder potentialities. Ph.D. Thesis, Mansoura University, Egypt

PARKER, C., AND J.D. FRYER. 1975. Weed control problems causing major reductions in world food supplies. FAO Plant Protection Bulletin, 23 (3): 8395.

PIELOU, E.C. 1969. An Introduction to Mathematical Ecology. Willey Interscience, New York, USA.

PIERCE, W.C., E.L. HAENISCH, AND D.T. SAWYER. 1958. Quantitative Analysis. Wiley Toppen, Tokyo.

PIMENTEL, D., L. LACH, R. ZUNIGA AND D. MORRISON. 2000. Environmental and economic costs of nonindigenous species in the United States. BioScience, 50 (1): 53-65.

PIPER, C.S. 1947. Soil and Plant Analysis. Interscience Publishers, Inc, New York, USA.

PIRIE, N.W. 1955. Protein. In: Peach K, Tracey VM (eds) Modern Methods of Plant Analysis. Springer Verlage, Berlin, pp 23- 68

SALAMA, F., M. ABD EL-GHANI AND N. ELTAYEH. 2013. Vegetation and soil relationships in the inland wadi ecosystem of central Eastern Desert, Egypt. Turkish Journal of Botany, 37: 489-498.

SAMUELSEN, A.B. 2000. The traditional uses, chemical constituents and biological activities of Plantago major L. A review. Journal of Ethnopharmacology, 71 (1): 1-21.

SHALABY, M.A. 1995. Studies on plant life at Kafr ElSheikh Province, Egypt. M.Sc. Thesis, Kafr ElSheikh,Tanta University

SHALTOUT, K.H., AND R.A. EL FAHAR. 1991. Diversity and phenology of weed communities in the Nile delta region. Journal of Vegetation Science, 2: 385-390.

SHALTOUT, K.H., A. SHARAF EL-DIN, AND R.A.
EL-FAHAR. 1992. Weed communities of the common crops in the Nile Delta region. Flora, 187 (5): 329-339.

SHEDED, M.G., AND Z.A. TURKI. 2000. Weed communities in south Nile delta. In: Proceeding of The $1^{\text {st }}$ International Conference on Biological Science (ICBC), Tanta, Egypt, Tanta University, pp 171-182

SHEHATA, M.N., AND R.A. EL-FAHAR. 2000. The vegetation of reclaimed areas in Salhya region. In: The $1^{\text {st }}$ International Conference on Biological Science, Tanta, Egypt, Tanta University, pp 315-332

SHELEY, R.L., T.J. SVEJCAR AND B.D. MAXWELL. 1996. A theoretical framework for developing successional weed management strategies on rangeland. Weed Technology, 10: 766-773.

SHUKLA, R.S., AND P.S. CHANDEL. 1989. Plant Ecology and Soil Science. S. Chand \& Company LTD. Ram Nagar, New Delhi, India.

SNEDECOR, G.W., AND W.G. COCHRAN. 1968. Statistical methods. 6 ed. The Iowa State University Press, USA.

SOTHERTON, N.W., N.D. BOATMAN, AND M.R.W. RANDS. 1989 The conservation headland experiment in cereal ecosystems. The Entomologist, 108 135-143.

STAVER, C., A. AGUILAR, V. AGUILAR, AND S. SOMARRIBA. 1995. Selective weeding: ground cover and soil conservation in coffee in Nicaragua. Institute for Low External Input Agriculture (ILEIA) Newsletter, 11 (3): 22-30.

TÄCKHOLM, V. 1974. Students' Flora of Egypt. 2nd ed. Cairo University Press., Cairo, Egypt.

TER BRAAK, C.J.F. 1987. The analysis of vegetationenvironment relationships by canonical correspondence analysis. Vegetatio, 69: 69-77.

VAN DELDEN, W., H. VAN DIJK AND K. WOLFF. 1992. Genetic strategies in Plantago. In: Kuiper PJC, Bos M (eds) Plantago: A Multidisciplinary Study, vol 89. Ecological Studies. Springer, Verlag, pp 295-310

WHITTAKER, R.H., AND S.A. LEVIN. 1977. The role of mosaic phenomena in natural communities. Theoretical Population Biology, 12 (2): 117-139.

ZIMDAHL, R.L. 2013. Fundamentals of Weed Science. 4th ed. Academic Press, San Diego, California. 


\section{دراسة بيئية علي ثلاث انواع من جنس البلانتاجو والنباتات المصاحبة في دلتا النيل بمصر \\ ابراهيم عبد الرحيم مشالي، احمد محمد عبد الجواد، محمد السيد ابو زياده، مها رجب دوييب

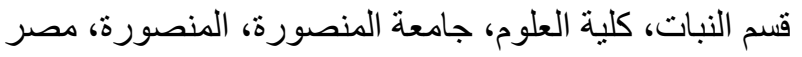

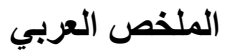

تلقي الدر اسة الحالية وصفا مفصلا عن بيئة ثلاثة أنواع من جنس البلانتاجو (Plantago) في مصر. وكانت اهداف هذا العمل كالاتي: 1) تحليل الغطاء النباتي لثنات مجتمعات من جنس البلانتاجو (نبات الودنة، لسان الحمل، البلانتاجو سكوروزا) في دلتا النيل في مصر باستخدام التحليل متعدد المتغيرات، 2) تحديد عوامل التربة التي تتحكم في وفرة وتوزيع المجتمعات النباتية المنعرف عليها في منطقة الدراسة، 3) تقييم ثراء وتنوع النباتي التي تنمو بشكل طبيعي في منطقة الدراسة. نم نوزيع مواقع

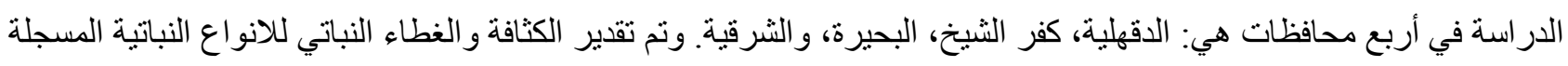
في 60 موقعاً وكذلك جمعت عينات تربة ممثلة وتم تحليلها لمعرفة خو اصها الفيزيائية و الكيميائية. وبتطبيق برنامج التحليل الدليلى ثنائي الإتجاه (TWINSPAN على البيانات الخاصة بوفرة الأنواع النباتية ممثلة بقيمة الاهمية و التى تم قياسها داخل 60 موقعاً بمنطقة الدراسة فقد أمكن تمييز أربع مجموعات نباتية وهي المجموعة "أ، ب" حيث سادهما نبات البلانتاجو سكوروزا بينما ساد

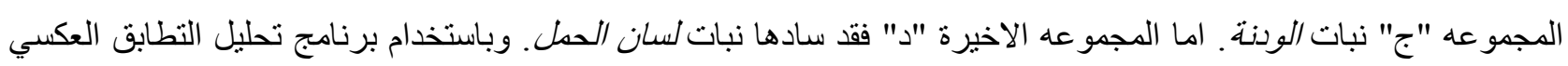
فقد وجد أن المجموعات النباتية قد فصلت بوضوح. وباستخدام برنامج تحليل التطابق الكنسى (DCA)

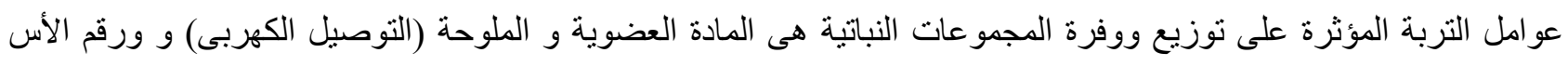

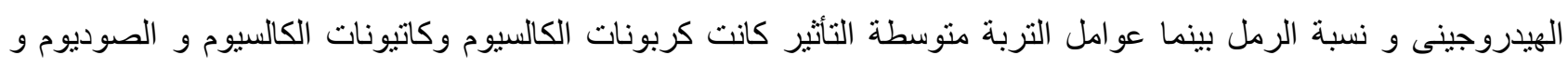
البوتاسيوم ونسبة الطمى والطين ومقدرة التربة على الاحتفاظ بالماء والفسفور الكلي الذائب. وقد تأثر مجتمع نبات البلانتاجو

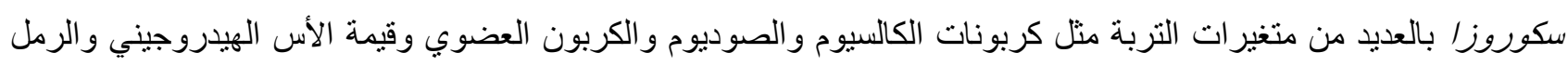

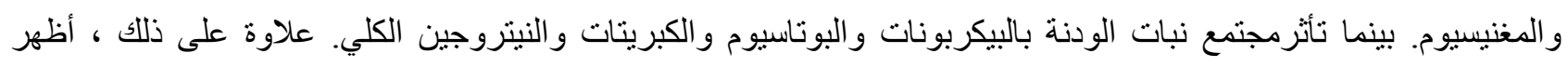
مجتمع نبات لسان الحمل علاقة وثيقة مع التوصيل الكهربائي وقدرة الاحتفاظ بالماء و الفوسفور الذائب الكلي و الطمي و الطين و المسامية. كما أظهرت قياسات التنوع البيولوجي أن مجتمع نبات لسان الحمل كان اكثر تتو عاوثر اءً، بينما أظهر مجتمع البلانتاجو سكوروز / أدنى تنوع وتو اجد ايضاً. 